\title{
Influence of the rock bridge in the roof of the developed space on the loading and deformation of stooks
}

\author{
Tamara Makeeva ${ }^{1, *}$, and Vitaly Trofimov ${ }^{2}$ \\ ${ }^{1}$ Moscow State University of Civil Engineering, Yaroslavskoe shosse, 26, 129337, Moscow, Russia \\ ${ }^{2}$ Institute of Comprehensive Exploitation of Mineral Resources Russian Academy of Sciences, \\ Kryukovsky tup., 4, 111020, Moscow, Russia
}

\begin{abstract}
The main regularities of deformation and loading of the stook during creation of the developed space with the great area of a side job are considered in the article. The possibility of formation of the set of balance among the stook barriers is estimated. The assessment of influence of rock "bridge" on the stook loading is given.
\end{abstract}

\section{Introduction}

In practice of construction of underground structures with great area of a side job, the maintenance of a roof is usually carried out by the means of basic elements. As a rule, those are the artificially built columns, or parts of the massif of rocks, left in the developed space (stooks). Stooks, as a rule, are the most loaded sites of the massif and therefore, they define working capacity, reliability and safety of functioning of all the mining design.

The loadings, operating on the stook, are usually defined within L.D. Shevyakov's hypothesis, which core is the statement that the greatest possible loading on the stook is in the varying degree, caused by the weight of all the rock mass up to the land surface. This concept has been borrowed held the ascendant position, when calculating the pressure upon the stook for many years, though some attempts became to consider unevenness of pressure upon the stook, located in the developed space.

The alternative approach is based on the hypothesis of balance arch. In this case, the existence of powerful barrier stooks, among which the balance arch is formed, is supposed. It is considered, that the stook located among the barrier stooks are in the favorable conditions as they are loaded only with the weight of rocks within the arch.

Deformation of the stook and the leaning massif are interdependent processes, which cannot be separated, and have to be considered within the uniform deformation model. The loading of the stook eventually defines the nature of their deformation and destruction.

Some research, connected with the determination of durability of the stook and ceiling in the underground developments are being conducted for many years, but the matter remains relevant for underground construction and mining industry.

\footnotetext{
*Corresponding author: makeeva13new@yandex.ru
} 
The first ratios for calculation of strength indicators of the stook in anthracitic mines of the USA have been received in the 19th century. Recently the great contribution to studying of the fundamental matters of geomechanics, connected with the assessment of the condition of the stook was brought by scientists of many countries of the world, whose research results are reflected in numerous publications. Later these results have been generalized in fundamental editions, to the devoted problems of geomechanics and, including, to calculation of stook and ceiling of the developed space [1-9].

The choice of parameters of the stook, i.e. their width and heights (and, respectively, width of chambers among the stooks) is connected with providing this or that mode of maintenance of the roof in various mining-and-geological conditions. Thus, when there are the water-bearing horizons in the massif of the leaning rocks, or there are the built-up territories on the day surface, there is the need of preservation of the integrity of the rock over the developed space at development of underground mining operations. Usually it is necessary to provide small deformations of all the leaning massif that is reached by leaving a massive stook in the developed space.

The common problem of the assessment of the state and behavior of the stook in traditional statement comes down to the solution of two tasks: the definition of the loadings operating on the stook and the determination of durability of a stook, depending on its geometrical sizes and strength characteristics of the material, composing the stook.

The first problem is usually solved within the Shevyakov's hypothesis, which is based on the statement, that the greatest possible loading on the stook is caused by the weight of all the rock mass to the land surface. If there is an infinite series of stooks, it does not raise any doubts because of the necessary power balance in the massif of rocks. However in case of final number of stooks such assumption is unevident and demands some justification [21].

However, the greatest attention has been paid to the solution of the second task, within which the set of experimental works, which results were embodied in many empirical ratios reflecting as a large-scale factor upon transition from the samples to the real entirely, and the factor of a form of a stook displayed by Zorn's coefficient (the relation of height of a stook to its width) has been performed.

Recently, unlike the initial stage of studying of the deformation processes in the stooks, essential attention is paid to accounting of various factors, complicating deformation process. That includes accounting of the increased pliability of the stook $[10,11]$ and their nonlinear deformation, accounting of pliability of the soil, heterogeneity of the leaning massif of rocks (existence of rocks bridges), ultra-boundary deformation of the rocks, composing the stook [12-16], etc. Some matters have found the solution and they were reflected in the corresponding methodical instructions, however, they are of interest for the researchers [1720] because of the lack of reasonable and adequate solutions.

The geological structure of rocks of the leaning massif is important. For sedimentary rocks, it usually has a layered structure (generally horizontal bedding). All these layers have various deformation and strength properties, which can be very often considered by the means of "effective" parameters, which are general for all the leaning massif. It is quite admissible, when the variation of parameters is small, i.e. the massif is almost uniform according to its deformation.

\section{General definition of the problem}

We will consider in details the mechanism of deformation and loading of the stook, when the leaning massif is uniform. Dredging of rocks with the formation of chambers leads to deformation of this massif, to its uneven subsidence. Besides, it is important to mean, that initially the massif is loaded as in the vertical, and horizontal directions with the initial squeezing tension increasing with the depth. 
Geomechanical assessment of the stook condition was carried out in the flat statement. The characteristic section and the corresponding parameters characterizing a geomechanical situation are presented in the figure 1.

When calculating was considered, that the developed space is at some depth $H$, which size during calculating is varied over a wide range, practically from 0 up to $1200 \mathrm{~m}$ with the corresponding initial tension, at the average density of leaning rocks $2110 \mathrm{~kg} / \mathrm{m}^{3}$. Horizontal initial tension was accepted as equal vertical, i.e. the coefficient of the side pressure is equal to 1 . This is quite acceptable assumption for sedimentary rocks. For definiteness, parameters of working-off were accepted as $a=6 \mathrm{~m}, b=4.75 \mathrm{~m}, L=\sim 100 \mathrm{~m}, m=10 \mathrm{~m}$. Deformation parameters of the containing rocks: $E=1 * 10^{10} \mathrm{~Pa}, \nu=0.31$.

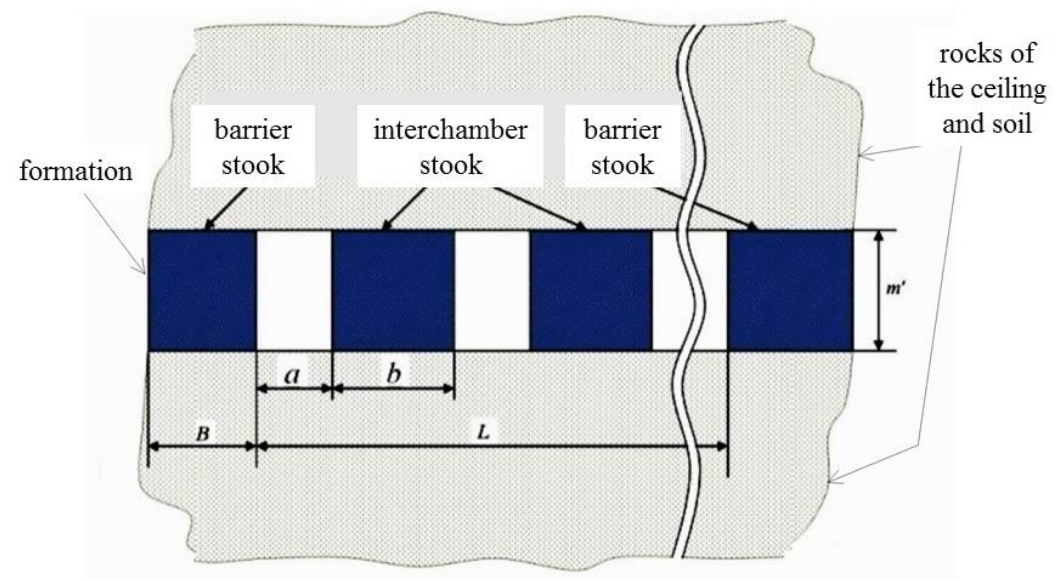

Fig. 1. Scheme of the system of stook in the developed space.

The problem is solved in the additional tension, thus, the boundary conditions are set on the surfaces of the formed chambers in the form of the normal tension, which is equal to the initial one, considered with a minus sign. After the calculation of fields of additional tension they are corrected by addition to them initial tension for obtaining values of full tension, which are actually acting in the massif. At the same time the received shifts during the problem definition in additional tension, are real.

\section{Numerical modelling}

We will start with the consideration of the question, what occurs, when there is one stook in the developed space, i.e. two chambers are fulfilled.

The distribution of the coefficient of concentration of vertical tension on the horizontal plane passing through the middle of a stook on its height is shown in the figure 2 . The zones of basic pressure with the maximum coefficient of concentration $\sim 1.3$ are formed on both sides of the developed space. At the same time, this maximum makes up $\sim 1.55$ on the stook.

This size, i.e. the maximum concentration, is characterizing the further loading of the stook though it is necessary to notice that the average size $\mathrm{k}$ on width of the stook is slightly less. Integrally the loading of the stook in this case is $\sim 1.5$. 


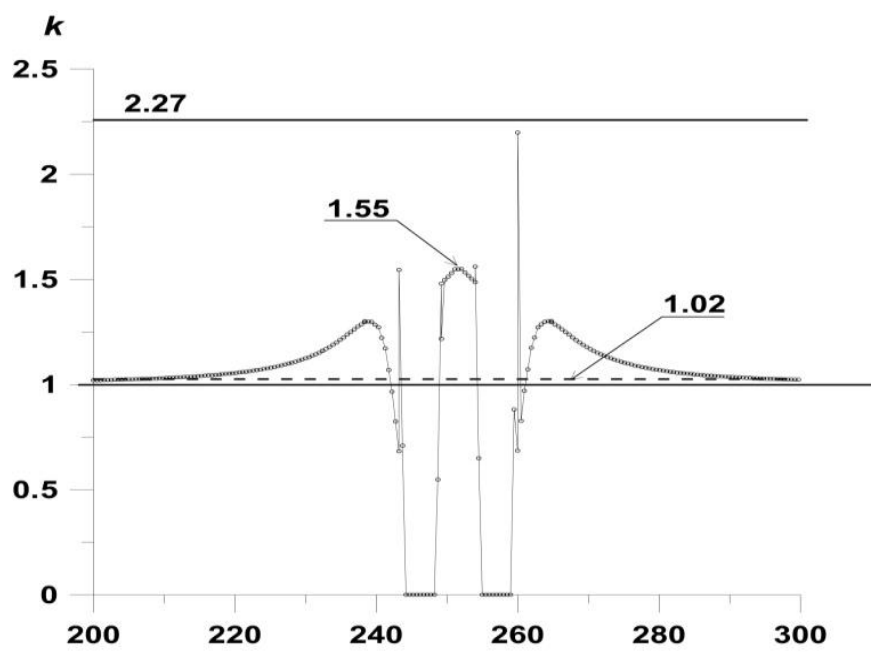

Fig. 2. Distribution of coefficient of concentration of vertical tension of $k$ on the horizontal line, a secant of a stook in half, in the presence in the developed space of one stook.

We should note that the average value $\mathrm{k}$ on all the interval $\mathrm{x}$ from $-\infty$ to $+\infty$ has to be equal 1 according to power balance of the massif before creation of the developed space. Numerical assessment of this size gives 1.02 with the divergence from theoretical value in $2 \%$. Probably, it is caused as the accuracy of a final element algorithm, which in particular, is connected with the advent of peaks of tension on border of the stook with chambers, and the sizes of settlement area limited in the horizontal direction (in this case the barrier stooks are absent).

It is easy to see, that concentration of vertical tension from the full weight of the leaning rocks is equal $\frac{a+b}{b}$ and for the chosen values of parameters of the stook and chambers makes 2.27. Thus, the loading of the stook if to judge according the maximum coefficient of concentration, is $\sim 70 \%$ from the greatest possible of the full weight of rocks. I.e. the stook is loaded not with the full weight of the leaning rocks, which part is redistributed on a regional part of layer. Nevertheless, the vertical squeezing tension on it are $\sim 1.55$ times are, than the initial tension.



Fig. 3. Coefficient of concentration of vertical tension $\mathrm{k}$ on the central stook, depending on the quantity of stooks within the block. 
Similar results are obtained, if we consider situations when there are several stooks in the developed space. For simplicity, we will consider a situation when the number of stooks is odd, i.e. the central stook is present, and that is convenient for interpretation of the results. At the same time, the size of coefficient of concentration depends on the number of stooks and increases with their increase. It is reflected in the figure 3 in which points have shown the dependence of the maximum coefficient of concentration of vertical tension on the central stook from quantity of stooks (a line is drawn for convenience of perception). The provided schedule allows to estimate the degree of proximity of loading of the stooks to its extreme size at the infinite number of stooks, which, obviously, is equal to 2.27.

Thus, during the development of mining operations, i.e. along with the increase in number of the fulfilled chambers there is a gradual increase of loading of the stook. As for the central stook, the increase of concentration of tension of $\mathrm{k}$ in the case under consideration can make up $30 \%$, from 1.55 to 2.27 .

We will consider regularities of loading of all the stooks within the fulfilled block. In the figure 4 the distribution of coefficient of concentration of vertical tension is shown of nine stooks, i.e. the maximum number for the set geometrical parameters.

In this case, as it has been done earlier, the averaging on all the medium surface gives size $\sim 1.08$, i.e. for $8 \%$ differing from theoretical because of the specified reasons.

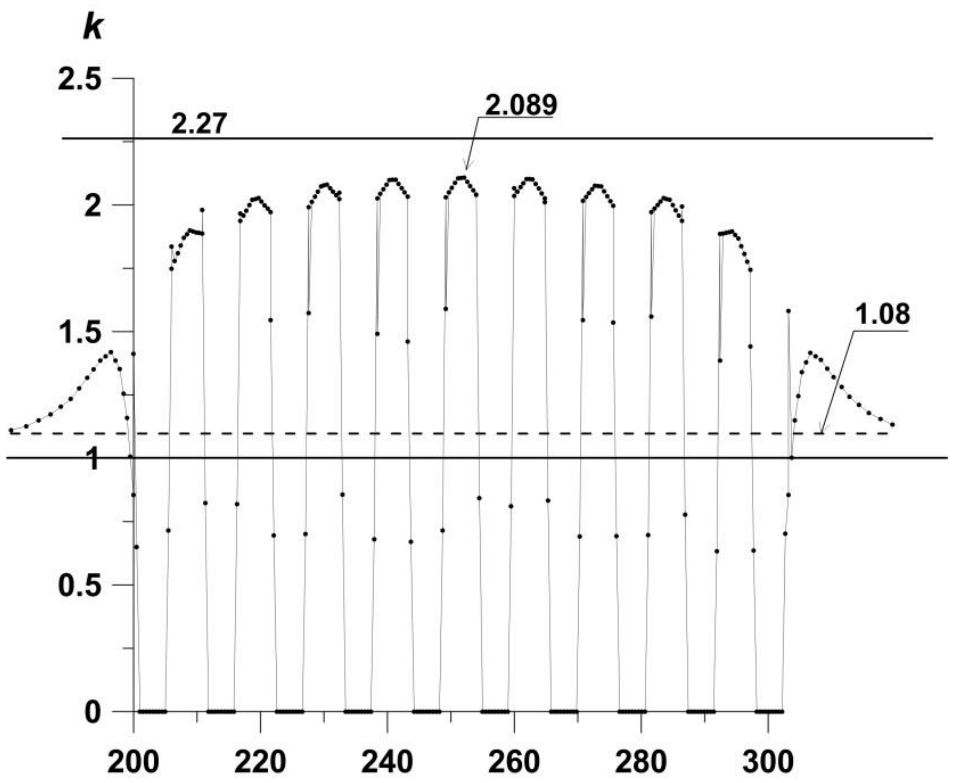

Fig. 4. Distribution of coefficient of concentration of vertical tension of $\mathrm{k}$ on the horizontal line, the secant of the stook in half, for nine stooks in the developed space.

In the figure 5 the maximum coefficient of concentration on nine stooks and in the zones of basic pressure is shown. The given curve is bending around for the curve in the figure 4 . As well as it was supposed the central stook is the most loaded one, and the least loaded stook is the next to the regional part of layer or to the barrier stook. The loading of all the stooks is much less than loading from the full weight of the leaning rocks that is 2.27 . At the same time, the squeezing vertical tension is practically more than twice exceeds the initial tension on all the stooks.

It is obvious, that in the absence of destruction of the stooks this curve is symmetrical for the central stook. Consideration of the situation, when there is an even number of stooks within the block, does not change an overall picture of their loading. The points, corresponding even entirely, also lay down on the presented curve. 
The curve, given in the figure 5 is approximated well by the ratio

$$
k=c-d|n-5|^{\alpha}
$$

which define the loading of each stook in the fulfilled block (fig. 5). At the chosen values of geometrical and deformation parameters we will have the following: $c=2.1, d=0.002, \alpha=3.23$.

At the same time, the size with is the coefficient of concentration of vertical tension for the central stook. It is obvious that it depends only on the ratio of width of the stook and span of the chamber.

We will also consider the deformation of the non-uniform leaning massif of rocks, when the option, of so-called, "rock bridge" is implemented.

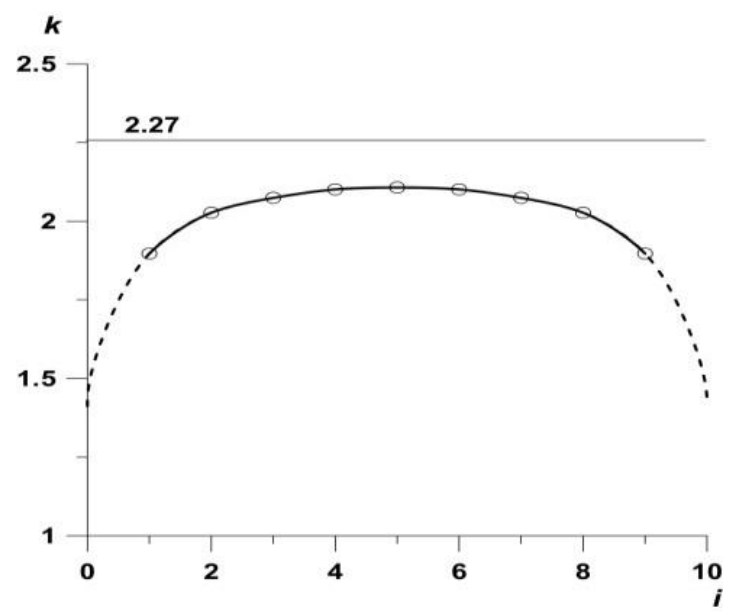

Fig. 5. Distribution of loading of the stook within the developed space for nine stooks.

In this case one of the layers, located over the developments has much higher deformation properties in comparison with other layers of the leaning rocks. At the same time, all the deformed massif in general becomes more rigid, and that leads to the reduction of displacement and redistribution of tension in it.

Further we will consider the main regularities of the new intense deformed condition of the massif and, in particular, the loading of the stook. We will consider that the rigid layer («bridge») settles down at the depth $\mathrm{Nl}$ from the day surface and its power is hl. Thus, the distance from the roof of the developed space to the soil of the layer makes

$$
\mathrm{d}=H-(H l+h l)
$$

We will carry out a series of calculations, in which the $H l$ and $h l$ will vary.

According to the results of calculations, we will control the sizes of the maximum subsidence of the roof of the developed space and the coefficient of concentration of vertical tension on the stooks. Comparing them to the corresponding sizes in the absence of "the rock bridge", it is possible to reveal the extent of the influence of the bridge on the condition of stooks.

In the conditions of the matter, we will vary $\mathrm{Nl}$ and $\mathrm{hl} \mathrm{so,} \mathrm{that} \mathrm{d}$ changed from 0 to, about $\mathrm{H}$. The deformation properties of rock of the "bridge" were accepted as Em $=1.1011 \mathrm{Pas}$, and $v_{M}=0.31$ respectively. Em is 10 times more than E for other rocks of the massif. Actually, Em is one more parameter of the matter, and it has to vary when calculating.

The results received are presented in figures 6,7 where the dependences of coefficient of concentration of vertical tension on the stook and the maximum lowering of a roof of the developed space for different $\mathrm{d}$ and $\mathrm{hl}$ are shown. 
We will note the characteristics of the received results for $\mathrm{k}$ and $\mathrm{v}$. All the curves for different $\mathrm{hl}$ in process of growth of $\mathrm{d}$ approach and at $\mathrm{d}$ are more than $\sim 40$ meters. At the same time the curves for $\mathrm{k}$ aspire to extreme size 1.548 (fig. 3), and for $\mathrm{v}$ they aspire to 0.0218. It means that the influence of "bridge", located further from the stook, does not depend on the distance from the layer to "rock bridge" and its power. In addition, k coincides with the coefficient of concentration of vertical tension on stook in the absence of "bridge".

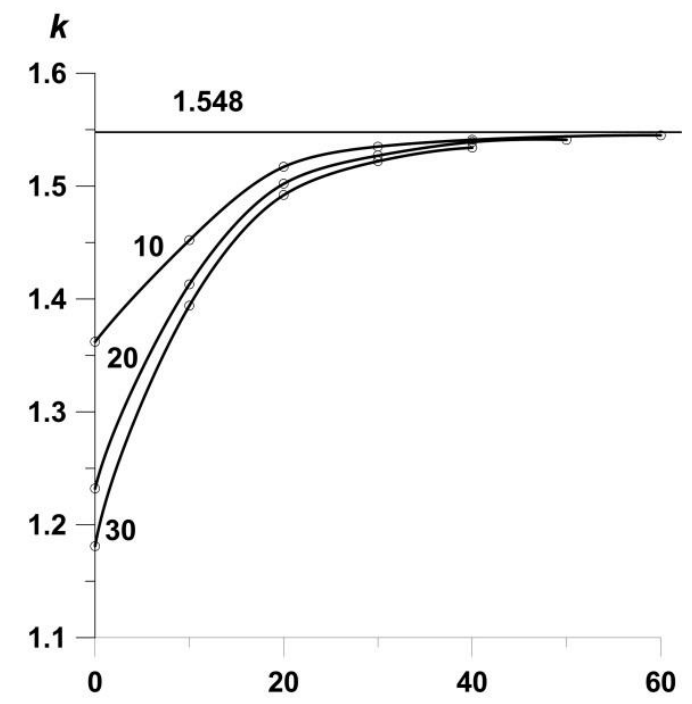

Fig. 6. Dependences of the coefficient of concentration of vertical tension on the stook from $\mathrm{d}$ and $\mathrm{hl}$.

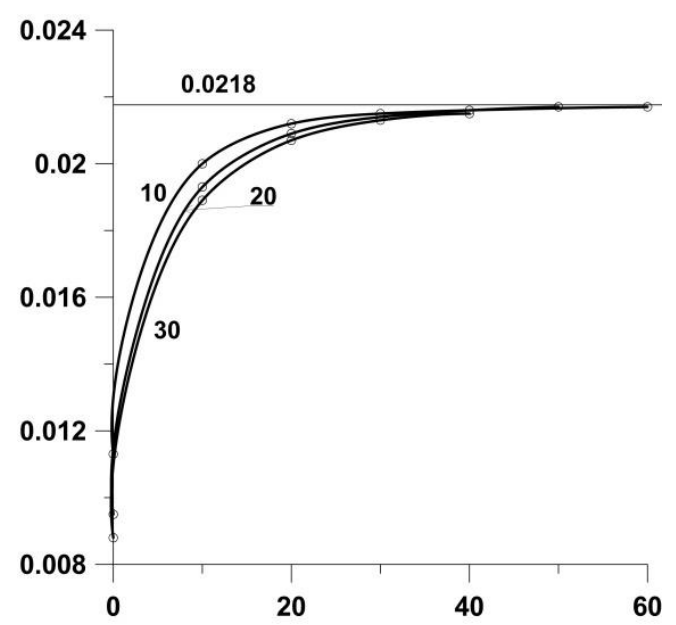

Fig. 7. Dependences of the maximum lowering of the roof of the developed space on $\mathrm{d}$ and hl.

Though $\mathrm{k}$ and $\mathrm{v}$ are dated for various points of the massif, it is possible to construct the correlation dependence between them. It is shown in the figure 8 .

We will note that all the curves, relating to various capacities of "the rock bridge" meet in one point.

In the figure 9 we combine the limit points of the convergence for curves of type of the stook, which are shown in the figure 8 , but which were received for different quantity of stooks $(1,3,5,9)$. Here the limit point for the infinite series of stooks is shown. We will note, 
that all the points (except the points for $\mathrm{d}=0$ ) lay down on the uniform straight line practically $k=0.55+44.35 v$.

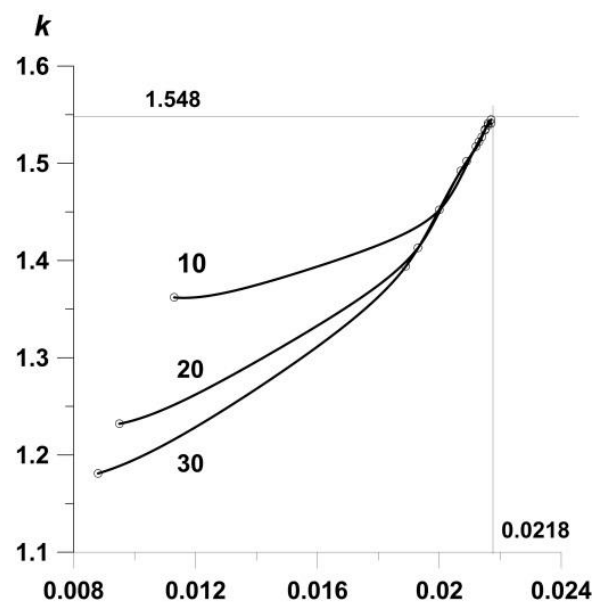

Fig. 8. Correlation dependences of coefficient of concentration of vertical tension in the stook from the maximum lowering of the roof of the developed space.

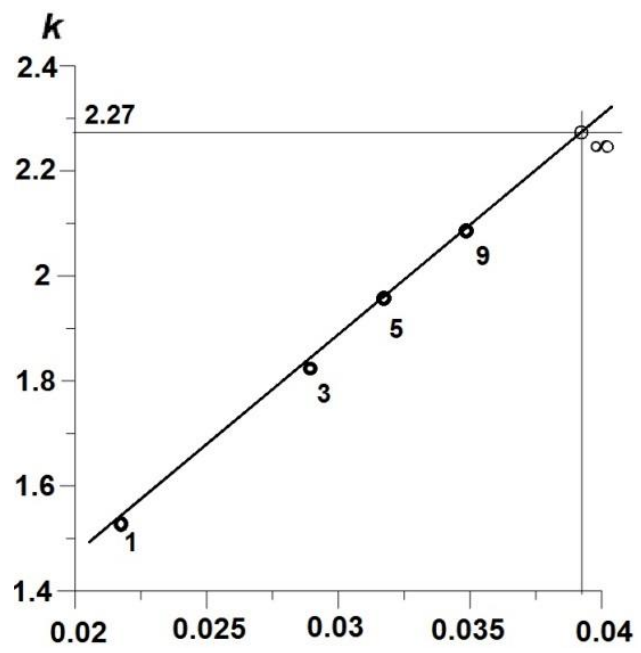

Fig. 9. Limit points of convergence for correlation curves of the stook, received for different quantity of the stooks $(1,3,5,9)$.

At the same time, it must be kept in mind that in the presence of several stooks, the curves of type shown in the figure 8 belong to the central stook.

\section{Conclusion}

The received result can be interpreted as though there was a fictitious arch in the massif and the stook would load only the rock in this arch, i.e. the loading was $\gamma \mathrm{Hc}$. At the same time $\mathrm{Hc} / \mathrm{H}$, i.e. the actual form of the "arch" as the percentage of depth $\mathrm{H}$, for each ith stook is also reflected the schedule in the figure 6 . It is connected with the existence of a regional part of layer, which (as well as the stook) receives additional loading in connection with the formation of the emptiness in the massif in the form of chambers. Considering an infinite series of the stook and chambers (nearly more than 50, see fig. 3), then in this case there are 
no regional parts of layer or they influence the distribution of tension a little, especially in the central part, and the following case is implemented: $k=2.27$.

The existence of pedigree "bridge" reduces the loading of the stook. At the same time, the power of "bridge" affects in a smaller measure, than its remoteness from the roof of the developed space. At some critical size of remoteness of the "bridge" ceases to exert any impact on loading of the stook. It means that the loading of the stook is formed generally by a part of the massif only in the direct vicinity of the developed the space as a result of its elastic restoration.

\section{References}

1. I.V. Baklashov, Deformation and destruction of pedigree massifs (Subsoil, Moscow, 1988)

2. Z. Benyavski, Management of mountain pressure (World, Moscow, 1990)

3. D.M. Bronnikov, N.F. Batches, G.I. Bogdanov, Development of ores at big depths (Science, Moscow, 1989)

4. Introduction to mechanics of rocky breeds (World, Moscow, 1983)

5. S.V. Smiths, V.N. Odintsev, M.E. Slonim, V.A. Trofimov, Metodologiya of calculation of mountain pressure (Science, Moscow, 1981)

6. Methods of determination of the sizes of basic stooks, (Academy, Moscow, 1962)

7. Modern problems of mechanics of rocks (Science, Leningrad, 1972)

8. I.M. Petukhov, A.M. Linkov, V.S. Sidorov, Calculation methods in mechanics of mountain blows and emissions (Subsoil, Moscow, 1992)

9. D.M. Kazikayev, Geomechanical processes at joint and repeated development of ores (Subsoil, Moscow, 1981)

10. N.V. Titov, Yu.V. Turuk, GIAB 8, 391-394 (2009)

11. K.N. Trubetskoy, Mountain messenger 4, 12-21 (1995)

12. K.N. Trubetskoy, FTPRPI 5, 3-16 (1995)

13. K.N. Trubetskoy, FTPRPI 5, 3-13 (1997)

14. K.N. Trubetskoy, Problems of mechanics of rocks. Works XI Russian conf. on fur. horn. Breeds $(\mathrm{SPb}, 1997)$

15. K.N. Trubetskoy, FTPRPI 2, 3-12 (1997

16. A.M. Grigoriev, GIAB 7, 205-211 (2008)

17. Methodical instructions by determination of the sizes of cameras and tselik by underground mining of ores of non-ferrous metals (Chita, 1988)

18. Methodical instructions by calculation of tselik of different function for conditions of fields of Pechenganikel plant (LISTEN, Leningrad, 1966)

19. Yu.G. Feklistov, A.D. Golotvin, Lithosphere 6, 130-135 (2015)

20. S. V. Bakumenko, S. V. Kuzmin, D. N. Demekhin, GIAB 7, 11-16 (2015) 